\title{
Prevalence of Salmonella spp. in broiler chicken flocks in northern Poland in 2014-2016
}

\author{
Dorota Witkowska ${ }^{1, A, C-F \oplus}$, Marta Kuncewicz ${ }^{1, B}{ }^{\text {, Joanna Paulina Żebrowska }}{ }^{1, B}{ }^{\circ}$, Jerzy Sobczak ${ }^{2, B} \oplus$, \\ Janina Sowińska ${ }^{1, E} \odot$ \\ 1 University of Warmia and Mazury, Olsztyn, Poland \\ ${ }^{2}$ Institute of Veterinary Hygiene, Olsztyn, Poland \\ A - Research concept and design, B - Collection and/or assembly of data, C - Data analysis and interpretation, \\ $D$ - Writing the article, E - Critical revision of the article, F- Final approval of article
}

Witkowska D, Kuncewicz M, Żebrowska JP, Sobczak J, Sowińska J. Prevalence of Salmonella spp. in broiler chicken flocks in northern Poland in 2014-2016. Ann Agric Environ Med. 2018; 25(4): 693-697. doi: 10.26444/aaem/99528

\section{Abstract}

Zoonotic serotypes of Salmonella enterica subsp. enterica are an important biological factor that poses a threat to public health, and broilers are often asymptomatic carriers of these bacteria. The aim of this study was to analyze the prevalence of Salmonella spp. and Salmonella serotypes in broiler chicken flocks in northern Poland in 2014-2016. The study was conducted on commercial flocks monitored by the Institutes of Veterinary Hygiene in Gdańsk and Olsztyn in 2014-2016. A total of 4,331 samples were investigated. Identification of Salmonella spp. was performed by a culture-dependent method followed by biochemical and serological confirmation tests, in accordance with Polish Standards. The total percentage of infected flocks in northern Poland reached $1.57 \%$. The prevalence of Salmonella spp. in broiler chickens decreased from $2.19 \%$ in 2014 to $1.22 \%$ in 2016. The percentage of flocks infected with S. Enteritidis and S. Typhimurium was $1.18 \%$ and $0.12 \%$, respectively (S. Enteritidis and S. Typhimurium were detected in $1.29 \%$ of flocks). The most common serotypes that had not been covered by the control programme were S. Mbandaka $(0.14 \%)$, S. Infantis $(0.07 \%)$ and, sporadically, S. Kentucky and S. Anatum. The percentage of infected flocks was higher (by $0.26 \%$ ) in the Pomeranian Region than in the Region of Warmia and Mazury, and it increased over the analyzed period - the highest number of positive samples were found in 2016 (2.04\%). In the Region of Warmia and Mazury, the percentage of infected flocks was highest in 2014 (3.08\%); in 2014-2016, the number of positive samples decreased steadily to reach $0.41 \%$ in 2016 . In the analyzed regions, the incidence of human salmonellosis was correlated with the percentage of infected broiler flocks.

\section{Key words}

Salmonella, broiler chicken, northern Poland

\section{INTRODUCTION}

Zoonotic serotypes of Salmonella enterica subsp. enterica are an important biological factor that poses a serious threat to public health $[1,2]$. Research indicates that broilers are often asymptomatic carriers of these bacteria [3]. In Poland, the first programme aimed at eliminating selected Salmonella serotypes from chicken farms was introduced 10 years ago (2007), and similar projects were implemented in successive years in laying hens (2008), broiler chickens (2010) and on turkey farms (2010). Despite the above, the reports of the European Food Safety Authority indicate that infections caused by Salmonella strains from poultry are still prevalent [4]. Poultry meat is the most common source of these bacteria, and human infections are often caused by cross-contamination or inadequate preparation of contaminated poultry meat intended for thermal processing $[1,4]$. According to Radkowski [3], the vast majority of chickens supplied to abattoirs are free of infection, but even a small percentage of Salmonella carriers on a farm is capable of cross-contaminating other birds during transport.

According to the most recent Eurostat data [6], Poland is the leading poultry producer in the EU. In 2014, Poland had a $13.9 \%$ share of the EU poultry market where broiler chicken

Address for correspondence: Dorota Witkowska, University of Warmia and Mazury Olsztyn, Poland

e-mail: dorota.witkowska@uwm.edu.pl

Received: 18.09.2018; accepted: 02.11.2018; first published: 29.11 .2018 meat is most widely consumed (79.8\%). Salmonella infections in poultry pose a serious and widespread problem because poultry consumption in Poland increased from $23.4 \mathrm{~kg}$ per capita in 2005 to $29.2 \mathrm{~kg}$ per capita in 2016 [7]. Human salmonellosis is most frequently caused by S. Enteritidis, S. Typhimurium, S. Infantis and S. Derby [4]. In the EU, the prevalence of human salmonellosis decreased significantly in 2008-2011, but the decreasing trend ended during 2012-2016, and the proportion of human salmonellosis cases remains constant [4]. Salmonellosis is the most ubiquitous zoonosis in Poland, and the number of confirmed human cases has increased in recent years [5]. According to the Institute of Agricultural and Food Economics, poultry consumption has grown steadily and is expected to increase in successive years due to its wide availability and low retail prices [8]. According to EFSA [4], national programmes aiming to reduce S. Enteritidis and S. Typhimurium infections in broiler chicken flocks in the EU have been successful because the prevalence target of $1 \%$ has been met in recent years. However, the notifications submitted to the Rapid Alert System for Food and Feed (RASFF), an effective tool for exchanging information between UE Member States about serious risks detected in relation to food and feed, indicate that Salmonella spp. was most frequently identified in poultry products in Poland in 2017 [9].

The aim of this study was to analyze the prevalence of Salmonella spp. and Salmonella serotypes in commercial flocks of broiler chickens in northern Poland in 2014-2016. 


\section{MATERIALS AND METHODS}

The study was conducted on commercial flocks of broiler chickens monitored by the Institutes of Veterinary Hygiene in Gdańsk and Olsztyn in 2014-2016, with the prior approval of these institutions for the use of laboratory results for scientific purposes. A total of 4,331 samples were investigated, 2,180 from Pomerania and 2,151 from Warmia and Mazury. Faecal samples for analyses were collected in accordance with the Instruction issued by the Chief Veterinary Officer [10]. Identification of Salmonella spp. on a weight basis/with surface faecal sampling swabs was performed by a culturedependent method followed by biochemical and serological confirmation tests, in accordance with the Salmonella spp. identification protocol (Edition 1 of 3 January 2014) and Polish Standards PN-EN ISO 6579:2003+A1:2007+AC:2014-11 and PN-EN ISO 6579:2003/AC:2014-11P [11]. The average percentage of samples collected and analyzed under the national control programme carried out by the General Veterinary Inspectorate was 5\% in the Region of Pomerania and 7\% in the Region of Warmia and Mazury. The remaining samples were collected by local veterinarians.

Samples were pre-enriched in buffered peptone water with a temperature of $18-27^{\circ} \mathrm{C}$, and incubated at $37^{\circ} \mathrm{C}\left( \pm 1^{\circ} \mathrm{C}\right)$ for 18 hours ( \pm 2 hours). Selective proliferation of Salmonella was carried out using the modified semi-solid Rappaport Vassiliadis (MSRV) medium (Oxoid CM1112B). The plates were incubated at a temperature of $41.5^{\circ} \mathrm{C}\left( \pm 1^{\circ} \mathrm{C}\right)$ for 24 hours ( \pm 3 hours). When negative results were noted, incubation was continued for another 24 hours ( \pm 3 hours). Salmonella colonies representing a normal growth pattern were transferred onto selective XLD (Oxoid CM0469B) and BGA (Oxoid CM0329B) media. Incubation was carried out at a temperature of $37^{\circ} \mathrm{C}$ $\left( \pm 1^{\circ} \mathrm{C}\right)$, and the results were read after 24 hours ( \pm 3 hours).

In the next step, biochemical confirmation tests were performed (PN-EN ISO-6579:2003, item 9.5.3) and Salmonella isolates were identified and serotyped by slide agglutination (PN-EN ISO-6579:2003, item 9.5.4) according to the White-Kauffmann-Le Minor scheme [12]. At least one typical or suspect colony was selected from each agar plate with a selective medium. Selected individual colonies were cultured on nutrient agar and soft agar plates. Pure cultures of Salmonella spp. were used in biochemical confirmation tests. The plates and cultures were incubated for 24 hours ( \pm 3 hours) at a temperature of $37^{\circ} \mathrm{C}\left( \pm 1^{\circ} \mathrm{C}\right)$, and serological identification of Salmonella isolates was performed.
The results of the analyses are shown in Table 1, including the total number of analyzed samples and the number of samples positive for S. Enteritidis and S. Typhimurium, and the remaining Salmonella serotypes. The percentage of infected broiler flocks in each month of 2014-2016, and the most common Salmonella serotypes identified in the analyzed regions, are presented graphically in Figures 1-3.

\section{RESULTS}

Table 1 presents the results of microbiological analyses for the presence of Salmonella spp. in broiler chicken flocks monitored by the Institutes of Veterinary Hygiene in Gdańsk and Olsztyn in 2014-2016. In the analyzed period, a total of 4,331 samples were collected in northern Poland, and positive results were noted in 68 cases $(1.57 \%)$. The prevalence of Salmonella spp. in broiler chickens decreased from 2.19\% in 2014 to $1.1 \%$ in 2015 and $1.22 \%$ in 2016 . The percentage of flocks infected with S. Enteritidis and S. Typhimurium was $1.18 \%$ and $0.12 \%$, respectively (S. Enteritidis and S. Typhimurium were detected in $1.29 \%$ of the flocks). The most common serotypes that had not been covered by the control programme were S. Mbandaka $(0.14 \%)$, S. Infantis $(0.07 \%)$ and, sporadically $(0.02 \%)$, S. Kentucky, S. Anatum and an unidentified Salmonella serotype. The percentage of infected flocks was higher (by $0.26 \%$ ) in the Region of Pomerania than in the Region of Warmia and Mazury, and increased over the analyzed period - the highest number of positive samples were found in 2016 (2.04\%). In the Region of Warmia and Mazury, the percentage of infected flocks was highest in 2014 (3.08\%); in 2014-2016, the number of positive samples decreased steadily to reach $0.41 \%$ in 2016 .

As shown in Figure 1 which illustrates the percentage of broiler flocks infected with Salmonella spp. in northern Poland in each month of 2014-2016, the percentage of positive samples was highest in September 2014 in the Region of Warmia and Mazury (12.1\%) and in December 2016 in the Region of Pomerania (9.4\%). In broiler flocks monitored by the Institute of Veterinary Hygiene in Olsztyn, Salmonella spp. were detected in 9 months in 2014, and in only 3 months in 2015 and 2016. In broiler flocks monitored by the Institute of Veterinary Hygiene in Gdańsk, positive samples were more evenly distributed across the months and years (5 months in 2014, and 6 months in 2015 and 2016).

Table 1. Prevalence of Salmonella spp. and Salmonella serotypes in broiler chicken flocks in northern Poland in 2014-2016

\begin{tabular}{|c|c|c|c|c|c|c|c|c|c|}
\hline \multirow{2}{*}{ Specification } & \multicolumn{4}{|c|}{ Pomerania } & \multicolumn{4}{|c|}{ Warmia and Mazury } & \multirow{2}{*}{ Total } \\
\hline & 2014 & 2015 & 2016 & 2014-16 & 2014 & 2015 & 2016 & 2014-16 & \\
\hline Number of analyzed samples & 704 & 742 & 734 & 2180 & 714 & 704 & 733 & 2151 & 4331 \\
\hline Number of positive samples & 9 & 13 & 15 & 37 & 22 & 6 & 3 & 31 & 68 \\
\hline Percentage of infected flocks (\%) & 1.278 & 1.752 & 2.044 & 1.697 & 3.081 & 0.852 & 0.409 & 1.441 & 1.570 \\
\hline S. Enteritidis & 8 & 10 & 9 & 27 & 17 & 5 & 2 & 24 & 51 \\
\hline S. Anatum & - & - & - & - & - & - & 1 & 1 & 1 \\
\hline S. Kentucky & - & - & - & - & - & 1 & - & 1 & 1 \\
\hline S. Infantis & - & - & 1 & 1 & 2 & - & - & 2 & 3 \\
\hline S. Mbandaka & 1 & 1 & 2 & 4 & 2 & - & - & 2 & 6 \\
\hline Other (S. sp.) & - & - & - & - & 1 & - & - & 1 & 1 \\
\hline
\end{tabular}




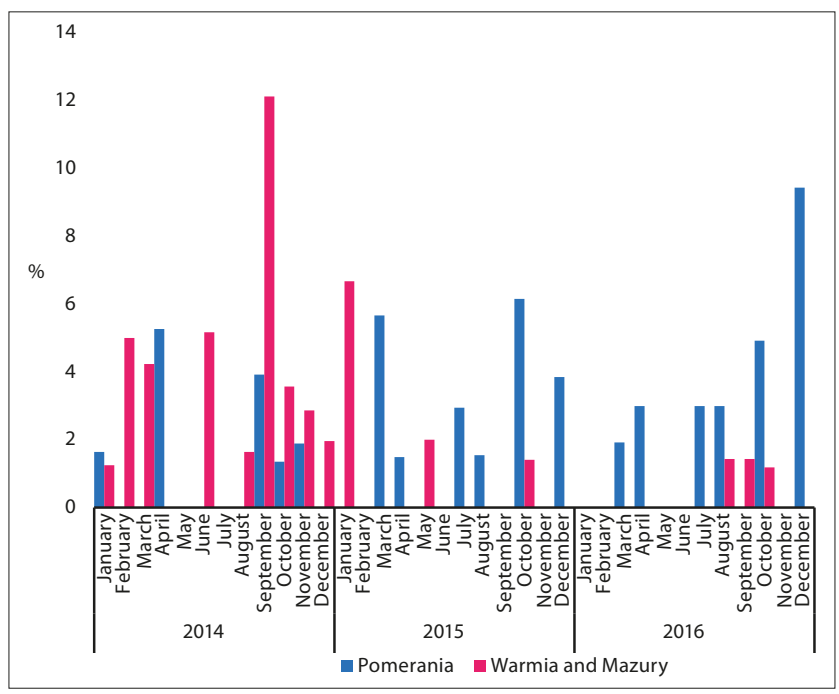

Figure 1. Percentage of broiler chicken flocks infected with Salmonella spp. in northern Poland in each month of 2014-2016

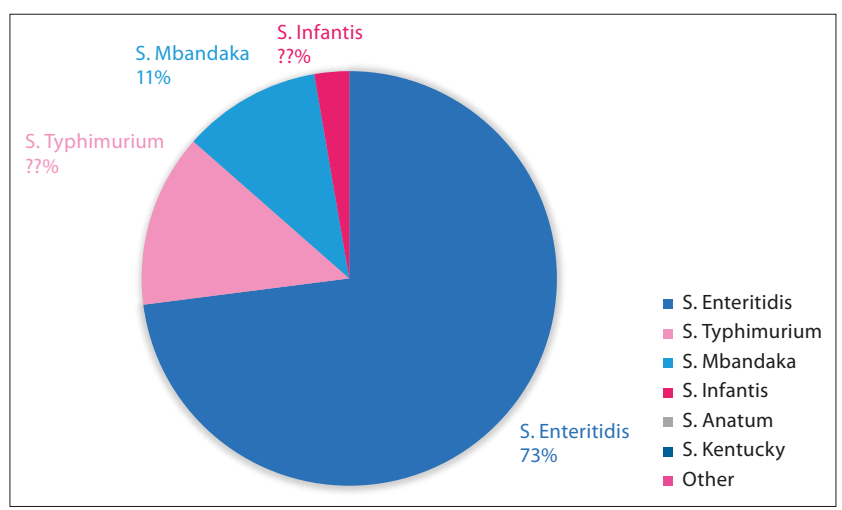

Figure 2. Salmonella serotypes identified in broiler chicken flocks in the Region of Pomerania in 2014-2016

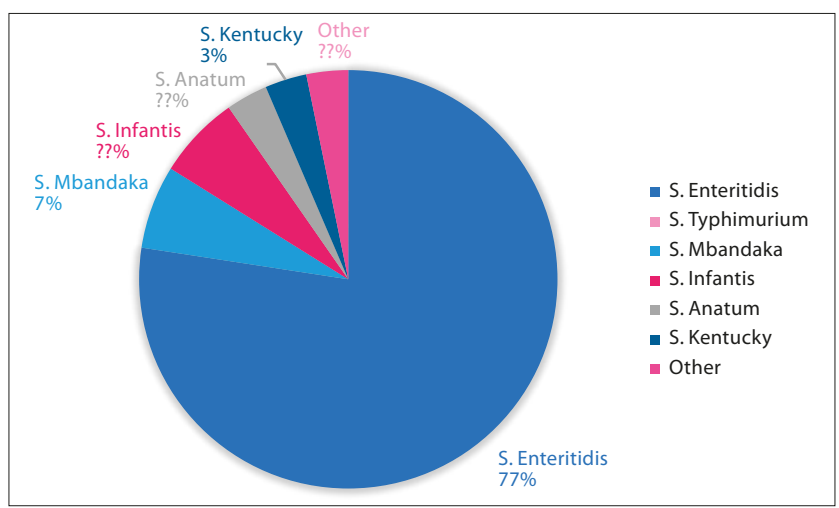

Figure 3. Salmonella serotypes identified in broiler chicken flocks in the Region of Warmia and Mazury in 2014-2016

A higher number of different Salmonella serotypes were identified in the Region of Warmia and Mazury. Salmonella Typhimurium was not detected in the Region of Warmia and Mazury, whereas in the Region of Pomerania, this serotype accounted for $13 \%$ of Salmonella-positive samples. In both regions, S. Enteritidis was the most common serotype in broiler flocks, accounting for 77\% in Warmia and Mazury and for $73 \%$ in Pomerania (Fig. 2-3).

\section{DISCUSSION}

According to $\mathrm{EU}[4,13,14]$ and domestic reports [5], Salmonella spp. are among the most ubiquitous pathogens that pose a serious risk for public health. At the primary production level, the prevalence Salmonella spp. in poultry flocks, including broiler chickens and turkeys, decreased or stabilized across the EU in 2016, compared with previous years, but the decreasing trend in laying hen flocks since the introduction of national control programsme in 2008 was reversed, and a significant increase in Salmonella detections was noted in 2015-2016 [4]. The results of the presented study (Tab. 1; Fig. 1) indicate considerable variations in the number of Salmonella detections across regions and years. The percentage of infected broiler chicken flocks generally decreased in northern Poland in 2014-2016. In the Region of Warmia and Mazury, the proportion of positive samples from broiler chicken farms decreased by $2.7 \%$ in $2014-2016$. In the Region of Pomerania, the counts of identified Salmonella bacteria in broiler farms increased by $0.8 \%$ between 2014 and 2016. This could be related to the seasonality of these bacteria and the differences between meteorological parameters in particular regions and years. Salmonella Enteritidis and Salmonella Typhimurium especially show strong associations with the weather conditions, for instance, high temperature [15]. On the other hand, the prevalence of Salmonella (Fig. 1) in flocks in Pomerania was the highest in December (2016), October (2015) and April (2014), whereas in Warmia and Mazury in September (2014) and January (2015). It should be underlined that the microclimate conditions in poultry buildings are maintained at optimal level throughout the whole year, therefore the dependence on outside temperature may be lower in the case of poultry flocks. Seasonality may be explained by a mixture of factors including, inter alia, climate, social, behavioural, agricultural, environmental and stochastic changes in immune populations [15]. The source of infections of Salmonella in poultry could be anything from a broad range of wild animals, including rats and mice [16]. In the autumn and winter seasons, rodents seek shelter in buildings, which may be the reason for the seasonality observed in the current study. Specialist chicken farms are usually closed units with a high level of bio-security. However, the occurrence of wild rodents may be difficult to exclude completely. Therefore, rodent control should be considered an important measure to provide good biosecurity on farms. [16].

In the EU [4], where the prevalence of Salmonella in broiler flocks reached $2.6 \%$ in 2016 and was considerably higher than in this study ( $1.6 \%$ on average), the prevalence increased by $0.4 \%$ in comparison with 2015 and decreased by $0.8 \%$ relative to 2014. In Poland, the percentage of infected broiler chicken flocks has been decreasing steadily [17]. A study conducted in 2005-2006 (two years before the introduction of control programmes) revealed that $58.7 \%$ of broiler flocks were infected with Salmonella spp. [18].

Commission Regulation (EU) No. 200/2012 concerning a Union target for the reduction of Salmonella Enteritidis and Salmonella Typhimurium in flocks of broiler chickens, as provided for in Regulation (EC) No. 2160/2003 of the European Parliament and of the Council [19], sets the target for the reduction of the maximum percentage of broiler flocks remaining positive for Salmonella Enteritidis and Salmonella Typhimurium, including monophasic Salmonella 
Typhimurium strains with the antigenic formula 1, 4, [5], 12:i:-, to $1 \%$ or less. This target has been achieved, and the prevalence of the above serotypes in Polish broiler flocks decreased between 2010 (S. Enteritidis and S. Typhimurium - $0.72 \%)$ and 2014 (0.15\%). The prevalence of S. Enteritidis and S. Typhimurium serotypes increased to $0.23 \%$ in 2015 [17], and it decreased to $0.1 \%$ in 2016 (the prevalence of S. Typhimurium was below $0.01 \%$ in $2014-2016$ ). In the EU, the prevalence of S. Enteritidis and S. Typhimurium serotypes also increased from $0.2 \%$ in 2014 to $0.26 \%$ in 2015 , and it reached $0.21 \%$ in $2016[4,13,14]$. The results obtained in the current study (Tab. 1) indicate that the reduction target has not yet been achieved in northern Poland, but the combined prevalence of S. Enteritidis and S. Typhimurium $(1.29 \%)$ in poultry flocks was only somewhat higher than the recommended value. In the Region of Pomerania, the proportion of S. Enteritidis positive samples increased from $1.14 \%$ in 2014 to $1.23 \%$ in 2016 , whereas the proportion of S. Typhimurium positive samples, which had not been detected in the region in 2014, was determined at $0.23 \%$ in 2015-2016. Salmonella Typhimurium was not detected in the Region of Warmia and Mazury in the discussed period, and the prevalence of S. Enteritidis decreased substantially from $2.38 \%$ in 2014 to $0.27 \%$ in 2016 . The number of S. Enteritidis positive samples in broiler chicken flocks decreased manyfold in Warmia and Mazury relative to 2001-2005 [20].

The aim of the control programme for broiler chicken flocks is to stabilize or decrease the prevalence of infections caused by the indicated Salmonella strains [17]. This is a very important consideration because the prevalence of foodborne diseases caused by Salmonella enterica serotype Enteritidis has increased significantly in the EU since 2006. This serotype is most frequently isolated from broiler chicken and laying hen meat [21]. Similarly to previous years, in 2016, the highest contamination with Salmonella enterica serotype Enteritidis was reported in poultry, in particular broiler chicken meat, intended for consumption after thermal processing. In contrast, the proportion of positive samples was negligent in fresh poultry meat. In 2016, the prevalence of $S$. Enteritidis also increased significantly in laying hens in the EU, and the relevant target has not been met. Poultry meat is often a source of S. Typhimurium (26\% in the EU in 2016) [4].

In Europe, Salmonella Enteritidis and S. Typhimurium have been the most common sources of human infections for many years. These serovars are responsible for the vast majority of human salmonellosis cases in Europe (70-80\% of infections caused by identified serotypes of Salmonella) [22]. In Poland, the proportion of S. Enteritidis serotypes that are most pathogenic for humans before the introduction of control programmes (in 2005/2006) was determined at $55.6 \%$, and the proportion of ST at 4.7\% [18]. According to Sadkowska-Todys and Czarkowski [23], in 2014, nearly 76\% of human salmonellosis cases in Poland were caused by the $\mathrm{S}$. Enteritidis serotype. The above authors also noted that in 2014, the number of salmonellosis cases had increased in Poland for the first time in many years. The epidemiological reports of the National Institute of Public Health - National Institute of Hygiene [24] revealed that the above trend stabilized in 2015-2016. An increase in the number of human salmonellosis cases was also observed in the Region of Pomerania where the disease incidence (number of new cases per 100,000 population) was determined at 21.6 in 2014 ,
23.4 in 2015 and 35.3 in 2016 [24]. In the presented study, the counts of identified Salmonella bacteria in broiler flocks also increased in the analyzed period (Tab. 1; Fig. 1). A similar correlation was noted between the prevalence of human salmonellosis and the number of Salmonella detections in broiler flocks in the Region of Warmia and Mazury (Tab. 1; Fig. 1). In the above region, the incidence of salmonellosis decreased from 25.7 in 2014 to 23.9 in 2015 and 19.5 in 2016. Human salmonellosis in Poland is the most common zoonosis, whereas in the EU Campylobacter infections occur most frequently (in recent years about 60\%) $[13,14]$ and over the last 10 years the incidence and prevalence of campylobacteriosis have increased in both the developed and developing countries of the world [25]. In Poland, the number of Campylobacter infections is significantly lower in comparison with Salmonella cases. In recent years (20142016), the prevalence of human salmonellosis was over $90 \%$ higher than campylobacteriosis [24, 13, 14]. According to epidemiological reports [24], the prevalence of human campylobacteriosis in the Region of Pomerania in 2014-2016 were significantly lower (77-90\%) than in the Region of Warmia and Mazury. Poultry is a major reservoir and source of transmission of campylobacteriosis to humans [25]. It is possible that the eradication of Salmonella rods from the chicken houses may promote the Campylobacter bacteria to colonize the emerging niche.

Similar to Europe, S. Enteritidis was the most common serotype in the analyzed regions of northern Poland [4, $13,14,22,23]$, and the percentage of detections reached $77 \%$ in the Region of Warmia and Mazury and 73\% in the Region of Pomerania (Fig. 2-3). Salmonella Typhimurium was not identified in Warmia and Mazury in 2014-2016, but it was detected in the Region of Pomerania. In recent years, S. Mbandaka and S. Infantis were identified in broiler flocks in both regions. These bacteria are less pathogenic, and they are not covered by the control programme, despite the fact $S$. Infantis is the third most common cause of human salmonellosis in Europe after S. Enteritidis and S. Typhimurium serovars. It should be noted that these bacteria are most frequently identified in broiler chicken flocks and broiler chicken meat. According to experts, S. Infantis could pose a growing threat to public health in the future due to high levels of drug resistance $[4,26]$. Rašeta et al. [27] isolated identical strains of S. Infantis from broiler carcasses and human salmonellosis patients. Single cases of S. Kentucky and S. Anatum were also reported in broiler flocks in the Region of Warmia and Mazury (Fig. 3). These findings should not be underestimated because Salmonella enterica serotype Kentucky has caused food poisoning in the EU in recent years [4]. Le Hello et al. [28] observed that the number of human infections with the multidrug-resistant S. Kentucky has increased significantly in many European countries and in the United States in recent years. In a study by Mąka et al. [29], selected Salmonella serotypes isolated from poultry, including S. Infantis and S. Typhimurium, were less prevalent, but were more frequently characterized by multidrug resistance than S. Enteritidis. In view of the above, according to some authors [28], serovars that are less virulent, but pathogenic and capable of colonizing poultry should be regularly monitored and controlled to minimize the risk for public health. To minimize this risk the effective bio-security measures should be implemented and developed incessantly on chicken farms. 


\section{CONCLUSIONS}

Salmonellainfections continue to affect broiler chickens despite the efforts made by international and domestic authorities and considerable improvement in the epidemiological status of poultry flocks after the implementation of Salmonella control programmes. In recent years, the prevalence of Salmonella has generally decreased in northern Poland, but considerable differences were observed between locations and years. The number of infected broiler chicken flocks has increased in the Region of Pomerania and decreased in the Region of Warmia and Mazury, and these observations are correlated with the incidence of human salmonellosis in these regions.

\section{REFERENCES}

1. Trawińska B, Saba L, Wdowiak L, Ondrašovičová O, NowakowiczDębek B. Evaluation of Salmonella rod incidence in poultry in the Lublin Province over the years 2001-2005. Ann Agric Environ Med. 2008; 15: 131-134.

2. Witkowska D, Mituniewicz T, Sowińska J, Wójcik A, Iwańczuk-Czernik K, Kleinszmidt K, et al. Occurrence of Salmonella in animal fodder in the Warmia and Mazury Province in 2001-2005. Med Wet. 2008; 64(8): 1062-1065.

3. Radkowski M, Zdrodowska B. Prevalence of Salmonella spp. in broiler chicken meat. Med Wet. 2016; 72(8): 516-519.

4. EFSA (European Food Safety Authority) and ECDC (European Centre for Disease Prevention and Control). The European Union summary report on trends and sources of zoonoses, zoonotic agents and foodborne outbreaks in 2016. EFSA J. 2017; 15(12): 5077, 228 pp.

5. National Institute of Public Health - National Institute of Hygiene. Reports on cases of infectious diseases, infections and poisoning in Poland; 2014-2017 http://wwwold.pzh.gov.pl (access: 2018.07.12).

6. EUROSTAT: Meat production statistics. 2017; http://ec.europa.eu/ eurostat/statistics-explained/index.php/Meat_production_statistics (access: 2018.07.05).

7. Central Statistical Office. Domestic deliveries and consumption of selected consumer goods per capita in 2016; https://stat.gov.pl (access: 2018.07.05).

8. Zawadzka D. The meat market status and perspectives. 2017; 52: 3-5.

9. Śledzińska K, Mąka Ł, Grochowska A, Pawłowska K, Windyga B, Karłowski K. Microbiological quality of food in the European Union countries based on RASFF notifications. Przem Spoż. 2010; 64: 32-34.

10. General Veterinary Inspectorate. Instruction of the Chief Veterinary Officer No. GIWpr-02010-9/2014 of 2 June 2014 on the measures to be taken by local veterinarians for controlling selected Salmonella serotypes in poultry flocks as provided for in Regulation (EC) No. 2160/2003 of the European Parliament and of the Council with regard to the national programs for controlling selected Salmonella serotypes in poultry flocks; http://wetgiw.gov.pl (access: 2018.06.28).

11. Polish Standard PN-EN ISO 6579: 2003. Food and feed microbiology. Horizontal detection of Salmonella spp.

12. Grimont PAD, Weill FX. Antigenic formulae of the Salmonella serovars. 9th ed. WHO Collaborating Center for Reference and Research on Salmonella. Pasteur Institute (Paris), 2007.
13. EFSA (European Food Safety Authority) and ECDC (European Centre for Disease Prevention and Control). The European Union summary report on trends and sources of zoonoses, zoonotic agents and foodborne outbreaks in 2014. EFSA J. 2015; 13(12): 4329, 190 pp.

14. EFSA (European Food Safety Authority) and ECDC (European Centre for Disease Prevention and Control). The European Union summary report on trends and sources of zoonoses, zoonotic agents and foodborne outbreaks in 2015. EFSA J. 2016; 14(12): 4634, 231 pp.

15. Cherrie MPC, Nichols G, Iacono GL, Sarran C, Hajat S, Fleming LE. Pathogen seasonality and links with weather in England and Wales: a big data time series analysis. BMC Public Health. 2018; 18(1): 1067. doi.org/10.1186/s12889-018-5931-6.

16. Backhans A, Fellström C. Rodents on pig and chicken farms - a potential threat to human and animal health. Infect Ecol Epidemiol. 2012; 2(1): 17093. doi: 10.3402/iee.v2i0.17093.

17. Regulation of the Minister of Agriculture and Rural Development of 18 January 2017 on the introduction of the national program for controlling selected Salmonella serotypes in broiler (Gallus gallus) flocks for 2017-2019. Journal of Laws, 2017, item 114.

18. Regulation of the Minister of Agriculture and Rural Development of 13 January 2014 on the introduction of the national program for controlling selected Salmonella serotypes in broiler (Gallus gallus) flocks for 2014-2016. Journal of Laws, 2014, item 92.

19. Commission Regulation (EU) No. 200/2012 of 8 March 2012 concerning a Union target for the reduction of Salmonella Enteritidis and Salmonella Typhimurium in flocks of broilers, as provided for in Regulation (EC) No. 2160/2003 of the European Parliament and of the Council (2012, OJ L 71, 9.3.2012, p. 31).

20. Mituniewicz T, Sowińska J, Iwańczuk-Czernik K, Witkowska D, Wójcik A, Kleinszmidt K, et al. Salmonella bacteria occurrence on poultry farms in Warmia and Mazury province between 2001-2005. Med Wet. 2017; 63(9): 1081.

21. Maćkiw E, Stasiak M, Kowalska J, Kucharek K. Microbiological hazards occurring in meat products. Przem Spoż. 2018; 72(3): 26-30.

22. Dera-Tomaszewska B, Kozłowski A. Statistical analysis of trend of human Salmonella infections in Poland in 1995-2007. Przegl Epidemiol. 2011; 65: 353-361.

23. Sadkowska-Todys M, Czarkowski MP. Salmonellosis in Poland in 2014. Przegl Epidemiol. 2016; 70(3): 358-366.

24. National Institute of Public Health -National Institute of Hygiene. Infectious diseases and poisoning in Poland (annual bulletin). 20142016; http://wwwold.pzh.gov.pl (access: 2018.07.12).

25. Kaakoush NO, Castaño-Rodríguez N, Mitchell HM, Ming Man S. Global epidemiology of Campylobacter infection. Clin Microbiol Rev. 2015; 28(3): 687-720.

26. Żebrowska JP, Witkowska D, Sobczak J, Mituniewicz T, Sowińska J. Occurrence of Salmonella on poultry farms, in flocks of pigeons and feed monitored by the Department of Veterinary Hygiene in Olsztyn in the years 2014-2015. Med Wet. 2017; 73(2): 111-117.

27. Rašeta M, Teodorović V, Bunčić $O$, Katić V, Branković-Lazić I, Polaček $\mathrm{V}$, et al. Antibiotic resistance and molecular studies on Salmonella enterica subspecies enterica serovar Infantis isolated in human cases and broiler carcasses. Acta Vet. 2014; 64: 257-268.

28. Le Hello S, Hendriksen RS, Doublet B, Fisher I, Nielsen EM, Whichard $\mathrm{JM}$, et al. International spread of an epidemic population of Salmonella enterica serotype Kentucky ST198 resistant to ciprofloxacin. J Infect Dis. 2011; 204: 675-684.

29. Mąka Ł, Maćkiw E, Ścieżyńska H, Pawłowska K, Popowska M. Antimicrobial susceptibility of Salmonella strains isolated from retail meat products in Poland between 2008 and 2012. Food Control. 2014; 6: 199-204. 